\title{
Effects of insulin detemir and NPH insulin on renal handling of sodium, fluid retention and weight in type 2 diabetic patients
}

\author{
K. V. Hendriksen • T. Jensen • P. Oturai • \\ B. Feldt-Rasmussen
}

Received: 19 July 2011 / Accepted: 27 September 2011 /Published online: 16 October 2011

(C) Springer-Verlag 2011

\begin{abstract}
Aims/hypothesis In type 2 diabetic patients, insulin detemir (B29Lys( $\varepsilon$-tetradecanoyl), desB30 human insulin) induces less weight gain than NPH insulin. Due to the proposed reduction of tubular action by insulin detemir, type 2 diabetic patients should have increased urinary sodium excretion, thereby reducing extracellular volume and body weight when changed from NPH insulin to insulin detemir. Methods In a randomised, open-labelled, two-way crossover study of 24 patients with type 2 diabetes, patients were first treated with NPH insulin or insulin detemir for 8 weeks. Thereafter, they were changed to the other insulin for 8 weeks. In a third 1 week period, they were changed back to the first insulin.

Results At the end of 8 weeks, body weight was reduced by $0.8 \pm 0.2 \mathrm{~kg}$ (mean $\pm \mathrm{SEM}$ ) on insulin detemir compared with NPH insulin $(p<0.01)$. After insulin detemir treatment, we also observed a significant reduction of lean body mass $(0.8 \pm 0.2 \mathrm{~kg}, p<0.05)$ and a non-significant reduction of extracellular volume $\left(0.8 \pm 0.5 \mathrm{l} / 1.73 \mathrm{~m}^{2}, p=0.14\right)$. The weight loss occurred after as early as 1 week $(0.8 \pm 0.2 \mathrm{~kg}$,
\end{abstract}

K. V. Hendriksen $\cdot$ B. Feldt-Rasmussen

Department of Nephrology, Rigshospitalet,

University of Copenhagen,

Copenhagen, Denmark

\section{T. Jensen $(\square)$}

Department of Endocrinology, Rigshospitalet,

Blegdamsvej 9,

DK-2100 København Ø, Denmark

e-mail: tonny.jensen@rh.regionh.dk

\section{P. Oturai}

Department of Clinical Physiology, Nuclear Medicine and PET, Rigshospitalet, University of Copenhagen,

Copenhagen, Denmark $p<0.001$ ), with a simultaneous and transient increase of urinary sodium excretion $(p=0.07)$.

Conclusions/interpretation Insulin detemir induces significant and sustained weight loss, which is first observed at 1 week after changing from NPH insulin. The initial weight loss seems to be related to changes in fluid volume and may reflect changed insulin action in the kidneys.

Trial registration: EudraCT 2008-001602-16; Clinical Trials.gov NCT00742976

Funding: The study was funded by a Libra Grant from NovoNordisk, DK.

Keywords Blood pressure · Body weight - Extracellular fluid volume - Type 2 diabetes . Urinary sodium excretion

\section{Abbreviations \\ DEXA Dual energy x-ray absorptiometry \\ NT-proBNP N Terminal-pro brain natriuretic peptide}

\section{Introduction}

Insulin detemir (B29Lys( $\varepsilon$-tetradecanoyl),desB30 human insulin) is a long-acting insulin analogue. Possibly due to its more stable, protracted absorption from the subcutaneous tissue, insulin detemir has been found to have a reduced risk of hypoglycaemia compared with NPH insulin [1,2]. However, the most constant finding in several studies has been the non-induction of weight gain by insulin detemir, in contrast to other insulins $[1,2]$. However, the mechanism underlying this weight advantage of insulin detemir relative to NPH insulin is not fully understood. Different mechanisms, such as (1) less snacking by patients due to a 
reduced fear of hypoglycaemia, (2) a more physiological role of insulin detemir in the liver or (3) stimulation of satiety centres in the hypothalamus have been proposed $[3,4]$.

Previous studies have shown that the antinatriuretic effects of insulin are most likely to be explained by increased tubular re-absorption of sodium [5] and that, cross-sectionally, there is a central role for sodium in the pathogenesis of blood pressure changes in diabetic patients [6].

The main site of insulin action in the kidney is the distal tubules. In acute insulin clamp and sodium infusion studies in humans, Skott and Stenvinkel showed increased fractional sodium re-absorption during hyperinsulinaemia [7, 8].

Due to the albumin-binding and hypothetically reduced glomerular filtration of insulin detemir, it can be hypothesised that tubular sodium re-absorption during insulin detemir treatment is reduced. To test the hypothesis that urinary sodium excretion is increased, and body weight and extracellular volume are reduced by insulin detemir, we compared the effects of insulin detemir and NPH insulin on the following predefined endpoints: body weight, extracellular fluid volume, sodium and water homeostasis, and body composition.

\section{Methods}

Patients We recruited 27 type 2 diabetic patients from the outpatient clinic at the Department of Endocrinology, Rigshospitalet; all patients were being treated with NPH insulin. Type 2 diabetes was defined according to the 1999 WHO criteria [9]. All patients had $\mathrm{HbA}_{1 \mathrm{c}}$ below $10 \%$ $(86 \mathrm{mmol} / \mathrm{mol})$ and normal urinary albumin excretion (i.e. $<30 \mathrm{mg}$ per $24 \mathrm{~h}$ ).

Exclusion criteria were any renal disease (serum creatinine $>130 \mu \mathrm{mol} / \mathrm{l}$ ), anaemia, uncontrolled hypertension (systolic blood pressure $>180 \mathrm{mmHg}$ and/or diastolic blood pressure $>95 \mathrm{mmHg}$ ), pregnancy or use of loop diuretics.

In the study group, 18 patients were taking an $\mathrm{ACE}$ inhibitor or an angiotensin II receptor antagonist, 12 of whom were also taking a thiazide diuretic. No changes were made in the doses of antihypertensive drugs during the study.

The protocol was approved by the Danish Medicine Agency and local ethics committee. The study was performed in accordance with the principles of the Declaration of Helsinki and written informed consent was obtained from each individual.

Study design After screening the patients continued their usual insulin treatment until, within 4 weeks, they were randomly assigned to two groups for a crossover study. Each patient then received NPH insulin or insulin detemir for 8 weeks. Subsequently, each patient switched to the other insulin treatment for 8 weeks. For a final 1 week period patients were again treated with the insulin used in the first period.

The patients were instructed to perform four-point blood glucose profiles weekly during the study. In the last week of each treatment period and on days 3 and 5 in the 1 st week after treatment change, the patients collected $24 \mathrm{~h}$ urine samples at home. In the same weeks, the patients twice conducted $24 \mathrm{~h}$ blood pressure measurements at home (90217 Ultralite Monitor; Spacelabs Healthcare, Medidane, Nærum, Denmark), namely on days 2 and 4.

Laboratory measurements Blood for laboratory measurements was collected from the antecubital vein without stasis. Thereafter, body composition was measured by dual energy X-ray absorptiometry (DEXA) whole-body scanner (Lunar Prodigy, GE Healthcare, Chalfont St Giles, UK). Total lean body mass and fat mass were measured. The GFR and plasma volume were measured over a period of $4 \mathrm{~h}$ by measuring the disappearance of ${ }^{51} \mathrm{Cr}$-labelled EDTA from plasma after a single intravenous injection of EDTA. Body weight was recorded with the same scales (MT32 type M5; Bisco scales, Farum, Denmark) for each patient (without coat and shoes) in the fasting state. Urinary sodium excretion rate was calculated as $U_{\mathrm{Na}} \times V$, where $U$ is units and $V$ is volume, and expressed as $\mathrm{mmol} / \mathrm{l}$.

$\mathrm{HbA}_{1 \mathrm{c}}$ was measured by a chromatographic technique (Tosoh, Tokio, Japan). Blood glucose was measured at home with an Ascensia Contour (Bayer Healthcare, Leverkusen, Germany). Urinary albumin concentration was measured by an immunoturbidimetric assay (Roche, Hvidovre, Denmark).

Plasma N terminal-pro brain natriuretic peptide (NTproBNP) was measured with a sandwich electrochemiluminescence immunoassay (Roche, Hvidovre, Denmark). The bioactive renin concentration was measured by a highsensitivity plasma renin activity assay (Glostrup Hospital, Glostrup, Denmark) and aldosterone was measured using a commercial kit (Coat-a-Count, catalogue item TKAL; Diagnostic Products, Los Angeles, CA, USA).

Statistical methods The results are expressed as means and SD when values were normally distributed, and as medians and interquartile ranges when the values were not normally distributed. Paired Student's $t$ tests were used when the values were normally distributed; otherwise, Wilcoxon's tests for paired differences were used. A two-tailed value of $p<0.05$ was considered to indicate statistical significance.

\section{Results}

Of the 27 patients recruited to the study, 24 completed both trial periods. Of the non-completers, one patient developed 
itching red skin reactions to insulin detemir at the injection site. In another patient blood glucose increased substantially despite increasing the dose of insulin detemir and the third non-completer stopped due to lack of compliance. Clinical characteristics of the 24 patients were: age $62 \pm 9$ years, body weight $93.1 \pm 15.1 \mathrm{~kg}$ and $\mathrm{HbA}_{1 \mathrm{c}} 7.6 \pm 1.2 \%(59 \pm$ $10 \mathrm{mmol} / \mathrm{mol}$ ) (all values given as mean $\pm \mathrm{SD}$ ).

Body weight and body composition Compared with NPH insulin treatment, insulin detemir treatment resulted in a significant reduction in body weight after 8 weeks $(p<0.05)$ (Table 1). At the same time, after insulin detemir treatment, a significant reduction of lean body mass $(0.8 \mathrm{~kg}, p<0.05)$ (Table 1) and a non-significant reduction of extracellular volume were demonstrated (Table 1). No difference in total fat mass was seen after the two treatment periods. Body weight was reduced after as early as 1 week of treatment with insulin detemir $(p<0.05)$ (Fig. 1 and Table 2), with all but two patients having reduced their body weight by this time-point.

Renal function, urinary sodium excretion and blood pressure Urinary sodium excretion was temporarily higher, although not statistically significant, at day 3 after changing from NPH insulin to insulin detemir and similar during both treatments after 5 days. GFR was not different after the two treatment periods. No differences were seen between $24 \mathrm{~h}$ blood pressure profiles measured at home on day 2 or day 4 (Table 2) after treatment change, or after 8 weeks on the two treatments (Table 1).

Metabolic and hormonal variables Plasma aldosterone, NT-proBNP and active renin were all similar after the two treatment periods (Table 1).

In the first week of the two treatments, the mean blood glucose, based on daily four-point preprandial blood glucose measurements, was similar (Table 2). During the following weeks the mean preprandial blood glucose was 0.6 to $1.2 \mathrm{mmol} / \mathrm{l}$ higher during insulin detemir treatment compared with NPH insulin. This corresponded to a slight increase in $\mathrm{HbA}_{1 \mathrm{c}}$, and occurred despite increasing the mean dose of insulin in the insulin detemir group from 55 to $65 \mathrm{U} /$ day.

\section{Discussion}

The major finding in the present study was the reduction of body weight after 1 and 8 weeks during treatment with insulin detemir in type 2 diabetic patients with normal urinary albumin excretion, compared with patients treated with NPH insulin. In addition, we demonstrated a significant reduction of lean body mass and a non-significant reduction of extracellular fluid volume after 8 weeks, as well as a non-significant transient increase of urinary sodium excretion in the first days of insulin detemir treatment.

The weight reduction observed after 8 weeks of treatment with insulin detemir is in accordance with previous observations $[1,2]$. However, the weight reduction of the same magnitude seen within 1 week of initiating insulin detemir is new and surprising. The reduced lean body mass and extracellular volume after insulin detemir treatment support the idea that insulin detemir exerts its initial effect on body weight, at least in part, by a reduced tendency to fluid retention compared with NPH insulin. This hypothesis is further supported by our demonstration of a (probably) transient increase in urinary sodium excretion in the first days after initiating insulin detemir treatment.

The changes in fluid homeostasis, although small, were not associated with changes in blood pressure. Blood
Table 1 Haemodynamic and metabolic indexes after 8 weeks on the two treatments

Results are shown as mean \pm SD unless otherwise indicated

${ }^{\mathrm{a}}$ Median (interquartile range)

${ }^{\mathrm{b}}$ Values below the detection limit of the assay in nine patients following insulin detemir and in 11 patients following NPH insulin treatment

\begin{tabular}{|c|c|c|c|}
\hline Variable & Insulin detemir & NPH insulin & $p$ value \\
\hline Body weight (kg) & $92.3 \pm 15$ & $93.3 \pm 15$ & $<0.01$ \\
\hline Extracellular fluid volume (1) & $18.2 \pm 3.4$ & $19.2 \pm 5.1$ & 0.14 \\
\hline Mean 24 h BP (mmHg) & $92 \pm 9$ & $91 \pm 8$ & NS \\
\hline GFR (ml/min) & $91.6 \pm 28.3$ & $92.3 \pm 29.6$ & NS \\
\hline Lean body mass $(\mathrm{kg})$ & $61.0 \pm 12.5$ & $61.8 \pm 12.8$ & $<0.05$ \\
\hline Fat mass $(\mathrm{kg})$ & $31.3 \pm 8.7$ & $31.4 \pm 8.1$ & NS \\
\hline Plasma NT-proBNP (pmol/l) $)^{\mathrm{a}, \mathrm{b}}$ & $6.2(<5.9-23.0)$ & $7.0(<5.9-17.4)$ & NS \\
\hline Plasma aldosterone $(\mathrm{pmol} / 1)^{\mathrm{a}}$ & $104(41-140)$ & $90(60-163)$ & NS \\
\hline Plasma active renin $(\mathrm{mIU} / \mathrm{l})^{\mathrm{a}}$ & $64(10-721)$ & $114(7-318)$ & NS \\
\hline $\mathrm{HbA}_{1 \mathrm{c}}(\%)$ & $8.2 \pm 1.3$ & $7.6(1.1)$ & $<0.01$ \\
\hline $\mathrm{HbA}_{1 \mathrm{c}}(\mathrm{mmol} / \mathrm{mol})$ & $66 \pm 11$ & $59(10)$ & $<0.01$ \\
\hline
\end{tabular}


Fig. 1 Individual body weight changes $(\mathrm{kg})$ during 1 week of treatment with insulin detemir (grey bars) and NPH insulin (black bars)

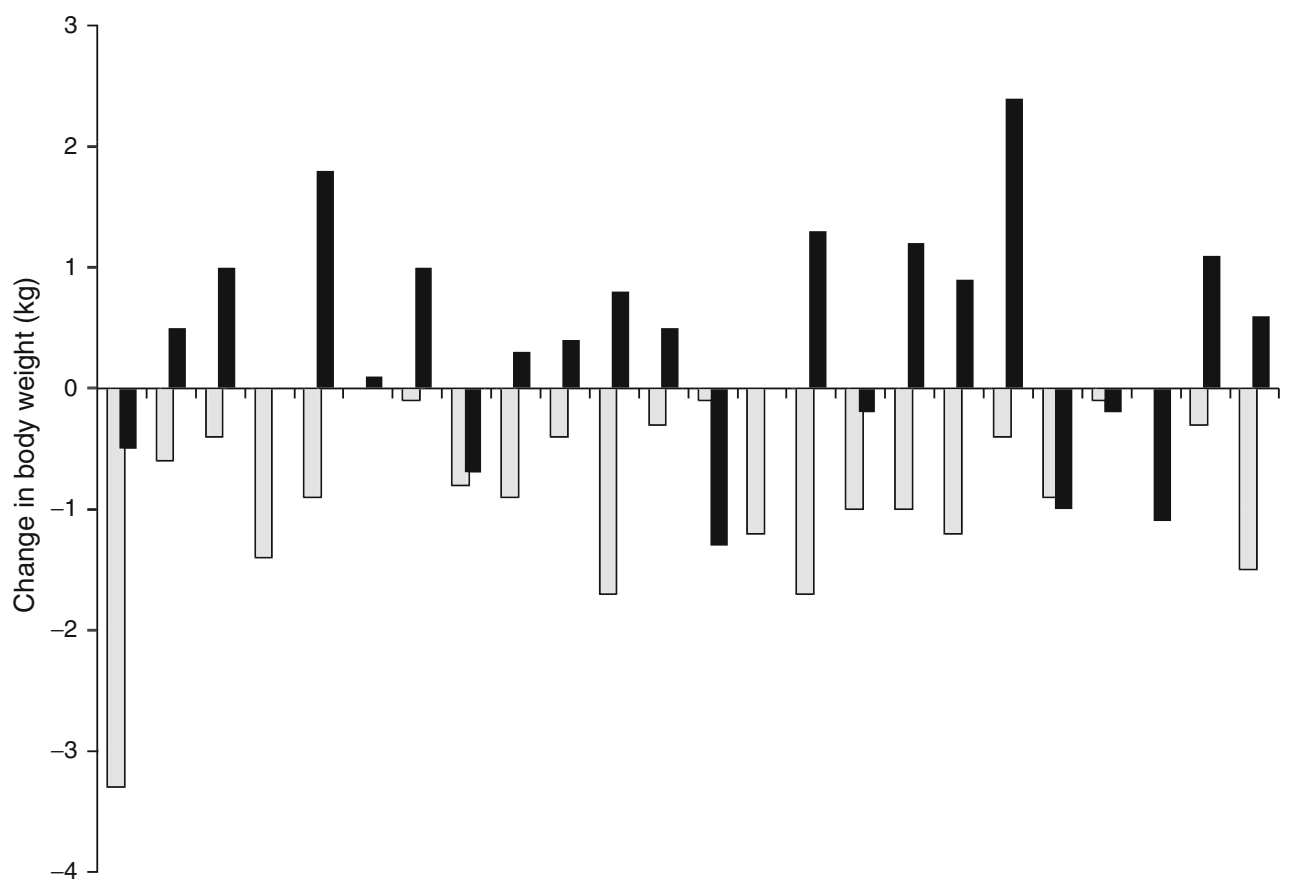

pressure was measured meticulously by $24 \mathrm{~h}$ measurements, conducted by patients at home, and we speculate that small changes in fluid volume are accompanied by regulatory mechanisms that attempt to keep blood pressure constant. Blood pressure was unchanged in the first week, and this might be explained by the time-point of blood pressure measurement, namely before and after the transient increase in urinary sodium excretion, thereby missing the time when a short decline in blood pressure might have been detected. Moreover, as patients were studied in the 1st week after the change in therapy, we cannot rule out some degree of carry-over effect of these intermediate-acting insulins. On the other hand, it has recently been demonstrated that glitazones, which significantly induced sodium retention, also did so without changing blood pressure [10].

We found a small, but significant increase in $\mathrm{HbA}_{1 \mathrm{c}}$ and a slight increase in insulin dose during treatment with insulin detemir. It is possible that the difference in metabolic control could, at least in part, explain the reduced fluid volume observed during insulin detemir treatment. However, it seems unlikely that a difference of $0.6 \%$ in $\mathrm{HbA}_{1 \mathrm{c}}$ would have any urinary osmotic effect, and indeed, we found no difference in urine volumes in the first days of the two treatments [11]. Moreover, mean blood glucose was similar in the 1st week of the two treatments, where the weight loss with insulin detemir occurred, another argument against the proposal above.

In conclusion, we found that insulin detemir had a weight-sparing effect compared with NPH insulin in normo-albuminuric type 2 diabetic patients. The effect was apparent after as early as 1 week of treatment, when a transient, although non-significant, increase in urinary sodium excretion was also demonstrated. Together with the reduction in extracellular fluid volume, these results support the idea that the reduced weight gain observed during insulin detemir treatment is, at least in part, explained by changes in fluid homeostasis.
Table 2 Urinary sodium excretion, body weight, $24 \mathrm{~h}$ blood pressure and blood glucose in the 1 st week of insulin detemir and NPH insulin treatments

Results are shown as mean \pm SD unless otherwise indicated

${ }^{\mathrm{a}}$ Median (interquartile range)

\begin{tabular}{llll}
\hline Variable & Insulin detemir & NPH insulin & $p$ value \\
\hline Urinary sodium excretion $(\mathrm{mmol} / \mathrm{l})^{\mathrm{a}}$ & & & \\
Day 3 & $212(160-266)$ & $185(126-245)$ & 0.07 \\
Day 5 & $191(148-222)$ & $178(145-250)$ & $\mathrm{NS}$ \\
Mean arterial pressure (mmHg) & & & \\
24 h BP day 2 & $90 \pm 8$ & $90 \pm 9$ & $\mathrm{NS}$ \\
24 h BP day 4 & $90 \pm 9$ & $90 \pm 9$ & $\mathrm{NS}$ \\
Body weight change day 7 (kg) & $-0.8 \pm 0.2$ & $0.4 \pm 0.2$ & $<0.01$ \\
Mean blood glucose (mmol/l) & 9.4 & 9.1 & $\mathrm{NS}$
\end{tabular}


Contribution statement KVH, TJ, BF-R and PO all contributed to the conception and design of the study and contributed to analysis and interpretation of data. All the authors participated in drafting and revising the article and all have approved the final version of the article.

Duality of interest The authors declare that there is no duality of interest associated with this manuscript.

\section{References}

1. Hermansen K, Davies M, Derezinski T, Ravn GM, Clauson P, Home P (2006) A 26-week, randomized, parallel, treat-to-target trial comparing insulin detemir with $\mathrm{NPH}$ insulin as add-on therapy to oral glucose-lowering drugs in insulin-naïve people with type 2 diabetes. Diabetes Care 29:1269-1274

2. Philis-Tsimikas A, Charpentier G, Clauson P, Ravn GM, Roberts VL, Thorsteinsson B (2006) Comparison of once-daily insulin detemir with NPH insulin added to a regimen of oral antidiabetic drugs in poorly controlled type 2 diabetes. Clin Ther 28:15691581

3. Russell-Jones D, Khan R (2007) Insulin-associated weight gain in diabetes: causes, effects and coping strategies. Diabetes Obes Metab 9:799-812
4. Hordern SV, Wright JE, Umpleby AM, Shojaee-Moradia F, Amiss J, Russell-Jones DL (2005) Comparison of the effects on glucose and lipid metabolism of equipotent doses of insulin detemir and NPH insulin with a 16-h euglycaemic clamp. Diabetologia 48:420-426

5. Nørgård K, Jensen T, Skøtt P et al (1991) Effect of insulin on renal haemodynamics and sodium handling in healthy subjects. Scand J Clin Lab Invest 51:367-376

6. Feldt-Rasmussen B, Mathiesen ER, Deckert T et al (1987) Central role for sodium in the pathogenesis of blood pressure changes independent of angiotensin, aldosterone and catecholamines in type 1 (insulin-dependent) diabetes mellitus. Diabetologia 30:610-616

7. Skott P, Hother-Nielsen O, Bruun NE et al (1989) Effects of insulin on kidney function and sodium excretion in healthy subjects. Diabetologia 32:694-699

8. Stenvinkel P, Bolinder J, Alvestrand A (1992) Effects of insulin on renal hemodynamics and the proximal and distal tubular sodium handling in healthy subjects. Diabetologia 35:1042-1048

9. WHO Consultation Report (1999) Definition, diagnosis and classification of diabetes mellitus and its complications. Part 1, pp 1-49

10. Zanchi A, Maillard M, Jornayvaz FR et al (2010) Effects of peroxisome proliferator-activated receptor (PPAR)-gamma agonist pioglitazone on renal and hormonal responses to salt in diabetic and hypertensive individuals. Diabetologia 53:1568-1575

11. Gerich JE (2010) Role of the kidney in normal glucose homeostasis and in the hyperglycaemia of diabetes mellitus: therapeutic implications. Diabet Med 27:136-142 